\title{
Extraction of optical solitons in birefringent fibers for Biswas-Arshed equation via extended trial equation method
}

https://doi.org/10.1515/nleng-2021-0011

Received Nov 25, 2020; accepted Apr 14, 2021.

\begin{abstract}
This article obtains optical solitons to the Biswas-Arshed equation for birefringent fibers with higher order dispersions and in the absence of four-wave mixing terms, in a media with Kerr type nonlinearity. Optical dark, singular and bright soliton solutions are articulated by applying an imaginative integration technique, the extended trial equation scheme. Various additional traveling wave solutions are produced with this integration technique, which include rational solutions, Jacobi elliptic function solutions and periodic singular solutions. From the mathematical analysis some constraints are recognized that ensure the actuality of solitons.
\end{abstract}

Keywords: Extended trial equation method; Birefringent fibers; Biswas-Arshed equation; Optical solitons

\section{Introduction}

Solitons play a pivotal role in almost every field of life and are prominently domineering in optics by virtue of their development and control in optical fibers and transmission of data across trans-continental distances. In the context of optical fibers the investigation of solitons is the conspicuous area of research in electrical engineering, telecommunication industry and applied sciences [1-13]. There is an extensive range of models that commendably describe the dynamics of formation of solitons such as the nonlinear Schrödinger's equation, FokasLenells equation, Kaup-Newell equation, Lakshmanan-

\footnotetext{
Muhammad Tahir, Aziz Ullah Awan, Department of Mathematics, University of the Punjab, Lahore 54590, Pakistan

*Corresponding Author: Kashif Ali Abro, Institute of Ground Water Studies, Faculty of Natural and Agricultural Sciences, University of the Free State, South Africa

Department of Basic Sciences and Related Studies, Mehran University of Engineering and Technology, Jamshoro, Pakistan, E-mail: kashif.abro@faculty.muet.edu.pk
}

Porsezian-Daniel model, complex Ginzburg-Landau equation, Gerdjikov-Ivanov equation, Kundu-Eckhaus equation, Radhakrishnan-Kundu-Lakshmanan equation [1425]. The propagation of solitons through optical fibers has been remain an engaging subject due its stunning applications in all optical communication systems. The area of telecommunication has experienced a remarkable development due to substantial progress in the industry of optical fibers. In recent few decades numerous results have been stated with polarization conserving fibers. Here we scrutinize the dynamics of solitons in the context of birefringent fibers where the pulses are polarized. Several investigations have been made for the extraction of optical solitons in birefringent fibers [26-34].

The Biswas-Arshed equation (BAE) reads [35]

$$
\begin{aligned}
& i \Omega_{t}+a_{1} \Omega_{x x}+a_{2} \Omega_{x t}+i\left(b_{1} \Omega_{x x x}+b_{2} \Omega_{x x t}\right) \\
& =i\left[\lambda\left(|\Omega|^{2} \Omega\right)_{x}+\mu\left(|\Omega|^{2}\right)_{x} \Omega+\theta|\Omega|^{2} \Omega_{x}\right],
\end{aligned}
$$

where $\Omega(x, t)$ represents the wave profile. In the recent works, this equation has engrossed a remarkable deliberation, see for Refs. [36-45], where the solitons are studied only along one component of this equation. Optical solitons for the BAE in birefringent fibers are investigated vie extended $\left(G^{\prime} / G\right)$-expansion method, modified simple equation method, trial equation scheme, exp-function method, auxiliary equation method, Sine-Cosine method and Jacobi elliptic function expansion approached [4652]. Additionally, the similar study on analytical solutions on different mathematical models involving fluid's mechanics [53-58], heat and mass transfer [59-65], nanofluids and nanoparticles [66-69], electrical instruments [70] and few other multi-dimensional models are adhered therein [71-72]. However, in this article the BAE will be investigated for optical solitons in birefringent fibers without four-wave mixing (4WM) terms with the aid of extended trial equation method. 


\section{Governing equation}

The coupled system obtained from (1) in birefringent fibers without 4WM reads [46]

$$
\begin{aligned}
i \mathfrak{p}_{t}+a_{1} \mathfrak{p}_{x x}+b_{1} \mathfrak{p}_{x t}+i\left(c_{1} \mathfrak{p}_{x x x}+d_{1} \mathfrak{p}_{x x t}\right) & =i\left[\lambda_{1}\left(\mathfrak{p}|\mathfrak{p}|^{2}\right)_{x}+y_{1}\left(\mathfrak{q}|\mathfrak{q}|^{2}\right)_{x}\right]+i\left[\beta_{1}|\mathfrak{q}|^{2}+\theta_{1}|\mathfrak{p}|^{2}\right] \mathfrak{p}_{x} \\
& +i\left[\alpha_{1}\left(|\mathfrak{q}|^{2}\right)_{x}+\mu_{1}\left(|\mathfrak{p}|^{2}\right)_{x}\right] \mathfrak{p}, \\
i \mathfrak{q}_{t}+a_{2} \mathfrak{q}_{x x}+b_{2} \mathfrak{q}_{x t}+i\left(c_{2} \mathfrak{q}_{x x x}+d_{2} \mathfrak{q}_{x x t}\right) & =i\left[\lambda_{2}\left(\mathfrak{q}|\mathfrak{q}|^{2}\right)_{x}+y_{2}\left(\mathfrak{p}|\mathfrak{p}|^{2}\right)_{x}\right]+i\left[\beta_{2}|\mathfrak{p}|^{2}+\theta_{2}|\mathfrak{q}|^{2}\right] \mathfrak{q}_{x} \\
& +i\left[\alpha_{2}\left(|\mathfrak{p}|^{2}\right)_{x}+\mu_{2}\left(|\mathfrak{q}|^{2}\right)_{x}\right] \mathfrak{q} .
\end{aligned}
$$

Here $b_{\ell}$ and $a_{\ell}$ are the coefficients of spatio-temporal dispersion (STD) and group velocity dispersion respectively, while $d_{\ell}$ and $c_{\ell}$ are the coefficients of third order STD and third order dispersion respectively, for $\ell=1$, 2. Next, $y_{\ell}$ and $\lambda_{\ell}$ stand for self-steepening terms, while the nonlinear dispersions are confirmed by $\beta_{\ell}, \theta_{\ell}, \alpha_{\ell}$ and $\mu_{\ell}$.

\subsection{Mathematical analysis}

To retrieve optical solitons of the coupled system (2), we utilize the traveling wave transformations

$$
\begin{aligned}
& \mathfrak{p}(x, t)=\mathfrak{Q}_{1}(\zeta) e^{i \phi(x, t)}, \\
& \mathfrak{q}(x, t)=\mathfrak{Q}_{2}(\zeta) e^{i \phi(x, t)},
\end{aligned}
$$

where

$$
\phi(x, t)=-\kappa x+\omega t+\epsilon \text { and } \zeta=x-v t .
$$

Here $\mathfrak{Q}_{\ell}(\zeta)$ is the amplitude, $v$ gives the soliton velocity, $\epsilon$ is the phase constant, $\kappa$ and $\omega$ are respectively the frequency and wave number of the soliton.

Implanting Eqs. (3)-(5) into (2) and dividing into imaginary and real parts, we attain

$$
\begin{gathered}
\left(c_{\ell}-d_{\ell} v\right) \mathfrak{Q}_{\ell}^{\prime \prime \prime}+\left(\kappa v b_{\ell}+\kappa^{2} v d_{\ell}-v-3 \kappa^{2} c_{\ell}+2 \kappa \omega d_{\ell}-2 \kappa a_{\ell}+b_{\ell} \omega\right) \mathfrak{Q}_{\ell}^{\prime} \\
-\left(2 \mu_{\ell}+\theta_{\ell}+3 \lambda_{\ell}\right) \mathfrak{Q}_{\ell}^{\prime} \mathfrak{Q}_{\ell}^{2}-2 \alpha_{\ell} \mathfrak{Q}_{l^{*}} \mathfrak{Q}_{l^{*}}^{\prime} \mathfrak{Q}_{\ell}-\beta_{\ell} \mathfrak{Q}_{\ell^{\prime}}^{\prime} \mathfrak{Q}_{l^{*}}^{2}-3 y_{\ell} \mathfrak{Q}_{l^{*}}^{\prime} \mathfrak{Q}_{l^{*}}^{2}=0,
\end{gathered}
$$

and

$$
\begin{array}{r}
\left(a_{\ell}-b_{\ell} v-2 v \kappa d_{\ell}+3 c_{\ell} \kappa-d_{\ell} \omega\right) \mathfrak{Q}_{\ell}^{\prime \prime}-\left(\omega+\kappa^{2} a_{\ell}+\kappa^{3} c_{\ell}-b_{\ell} \kappa \omega-\kappa^{2} d_{\ell} \omega\right) \mathfrak{Q}_{\ell} \\
-\kappa\left(\lambda_{\ell}+\theta_{\ell}\right) \mathfrak{Q}_{\ell}^{3}-\beta_{\ell} \kappa \mathfrak{Q}_{l^{*}}^{2} \mathfrak{Q}_{\ell}-y_{\ell} \kappa \mathfrak{Q}_{l^{*}}^{3}=0,
\end{array}
$$

where $\ell^{\star}=3-\ell$ and $\ell=1,2$. The balancing principle suggests that $\mathfrak{Q}_{l^{*}}=\mathfrak{Q}_{\ell}$.

Therefore, from Eq. (6) we have

$$
\begin{array}{r}
\left(c_{\ell}-v d_{\ell}\right) \mathfrak{Q}_{\ell}^{\prime \prime \prime}+\left(\kappa b_{\ell} v+\kappa^{2} d_{\ell} v-v-3 \kappa^{2} c_{\ell}+2 \kappa \omega d_{\ell}-2 \kappa a_{\ell}+b_{\ell} \omega\right) \mathfrak{Q}_{\ell}^{\prime} \\
-\left(2 \mu_{\ell}+\theta_{\ell}+3 \lambda_{\ell}+2 \alpha_{\ell}+\beta_{\ell}+3 y_{\ell}\right) \mathfrak{Q}_{\ell}^{\prime} \mathfrak{Q}_{\ell}^{2}=0 .
\end{array}
$$

From Eq. (8), we attain

$$
v=\frac{3 \kappa^{2} c_{\ell}-2 \kappa \omega d_{\ell}+2 \kappa a_{\ell}-b_{\ell} \omega}{b_{\ell} \kappa+d_{\ell} \kappa^{2}-1} \text { and } v=\frac{c_{\ell}}{d_{\ell}}
$$

along with the restraint conditions

$$
2 \mu_{\ell}+\theta_{\ell}+3 \lambda_{\ell}+\beta_{\ell}+2 \alpha_{\ell}+3 y_{\ell}=0, \quad \ell=1,2 .
$$

Comparing the two values of $v$ in (9), leads to the restraints

$$
3 c_{\ell} d_{\ell} \kappa^{2}-2 \kappa \omega d_{\ell}^{2}+2 d_{\ell} a_{\ell} \kappa-\omega d_{\ell} b_{\ell}-\kappa c_{\ell} b_{\ell}-\kappa^{2} d_{\ell} c_{\ell}+c_{\ell}=0, \quad \ell=1,2 .
$$

Now, from Eq. (7), we have

$$
\begin{array}{r}
\left(a_{\ell}-b_{\ell} v-2 \kappa d_{\ell} v+3 c_{\ell} \kappa-d_{\ell} \omega\right) \mathfrak{Q}_{\ell}^{\prime \prime}-\left(\omega+\kappa^{2} a_{\ell}+\kappa^{3} c_{\ell}-\omega b_{\ell} \kappa-\kappa^{2} d_{\ell} \omega\right) \mathfrak{Q}_{\ell} \\
-\kappa\left(\lambda_{\ell}+\theta_{\ell}+\beta_{\ell}+y_{\ell}\right) \mathfrak{Q}_{\ell}^{3}=0,
\end{array}
$$

where $\ell=1$, 2. Eq. (12) will now be scrutinized by extended trial equation method. 


\subsection{Application of extended trial equation method}

In this subsection, we employ the extended trial equation technique $[7,8]$ to Eq. (12) for constructing the exact solutions of the system (2).

Case-1. The solution of Eq. (12) can be expressed as

$$
\mathfrak{Q}_{\ell}=\sum_{i=0}^{\varrho} \delta_{i} \Psi^{i},
$$

where $\delta_{i}$ are unknown constant to be determined such that $\delta_{\varrho} \neq 0$ and

$$
\left(\Psi^{\prime}\right)^{2}=\Lambda(\Psi)=\frac{\Gamma(\Psi)}{\Omega(\Psi)}=\frac{\eta_{\sigma} \Psi^{\sigma}+\cdots+\eta_{1} \Psi+\eta_{0}}{\chi_{\rho} \Psi^{\rho}+\cdots+\chi_{1} \Psi+\chi_{0}},
$$

where $\eta_{0}, \ldots, \eta_{\sigma}$ and $\chi_{0}, \ldots, \chi_{\rho}$ are arbitrary constants to be identified such that $\eta_{\sigma} \neq 0$ and $\chi_{\rho} \neq 0$. Eq. (14) can be transformed into an integral form as:

$$
\pm\left(\zeta-\zeta_{0}\right)=\int \frac{d \Psi}{\sqrt{\Lambda(\Psi)}}=\int \sqrt{\frac{\Omega(\Psi)}{\Gamma(\Psi)}} d \Psi .
$$

The balancing process reveals that

$$
\sigma=\rho+2 \varrho+2 .
$$

By assuming $\sigma=4, \varrho=1$ and $\rho=0$ in (16), we arrive at

$$
\mathfrak{Q}_{\ell}=\delta_{0}+\delta_{1} \Psi .
$$

Implanting Eq. (17) along with Eq. (14) into (12) and evaluating the resultant system of equations, we attain

$$
\begin{gathered}
\eta_{0}=\eta_{0}, \quad \eta_{1}=\eta_{1}, \quad \chi_{0}=\chi_{0}, \quad \delta_{0}=\delta_{0}, \quad \delta_{1}=\delta_{1}, \\
\omega=\frac{\eta_{1} \delta_{1}\left(a_{\ell}+3 c_{\ell} \kappa-b_{\ell} v-2 d_{\ell} \kappa v\right)-2 \kappa \delta_{0} \chi_{0}\left\{a_{\ell} \kappa+c_{\ell} \kappa^{2}+\delta_{0}^{2}\left(\theta_{\ell}+\lambda_{\ell}+\beta_{\ell}+y_{\ell}\right)\right\}}{\eta_{1} \delta_{1} d_{\ell}-2 \delta_{0} \chi_{0}\left(b_{\ell} \kappa+d_{\ell} \kappa^{2}-1\right)}, \\
\eta_{2}=\frac{4 \kappa \delta_{0}^{3} \chi_{0}\left(\theta_{\ell}+\lambda_{\ell}+\beta_{\ell}+y_{\ell}\right)\left\{\kappa\left(b_{\ell}+d_{\ell} \kappa\right)-1\right\}+\eta_{1} \delta_{1}\left\{a_{\ell}\left(b_{\ell} \kappa-1\right)+b_{\ell} v-k_{1} \kappa\right\}}{-2 \delta_{0}\left(a_{\ell}-a_{\ell} b_{\ell} \kappa-b_{\ell} v+k_{2} \kappa\right)}, \\
\eta_{3}=\frac{\kappa \delta_{1}\left(\theta_{\ell}+\lambda_{\ell}+\beta_{\ell}+y_{\ell}\right)\left\{\eta_{1} \delta_{1} d_{\ell}-2 \delta_{0} \chi_{0}\left(b_{\ell} \kappa+d_{\ell} \kappa^{2}-1\right)\right\}}{a_{\ell}-a_{\ell} b_{\ell} \kappa-b_{\ell} v+k_{2} \kappa}, \\
\eta_{4}=\frac{\kappa \delta_{1}^{2}\left(\theta_{\ell}+\lambda_{\ell}+\beta_{\ell}+y_{\ell}\right)\left\{\eta_{1} \delta_{1} d_{\ell}-2 \delta_{0} \chi_{0}\left(b_{\ell} \kappa+d_{\ell} \kappa^{2}-1\right)\right\}}{4 \delta_{0}\left(a_{\ell}-a_{\ell} b_{\ell} \kappa-b_{\ell} v+k_{2} \kappa\right)},
\end{gathered}
$$

where

$$
\begin{aligned}
& k_{1}=b_{\ell}^{2} v+3 c_{\ell}-3 b_{\ell} \kappa\left(c_{\ell}-d_{\ell} v\right)+2 d_{\ell}^{2} \kappa^{2} v-2 c_{\ell} d_{\ell} \kappa^{2}-2 d_{\ell} v+3 \delta_{0}^{2} d_{\ell}\left(\theta_{\ell}+\lambda_{\ell}+\beta_{\ell}+y_{\ell}\right), \\
& k_{2}=b_{\ell}^{2} v+3 c_{\ell}-3 b_{\ell} \kappa\left(c_{\ell}-d_{\ell} v\right)+2 d_{\ell}^{2} \kappa^{2} v-2 c_{\ell} d_{\ell} \kappa^{2}-2 d_{\ell} v+\delta_{0}^{2} d_{\ell}\left(\theta_{\ell}+\lambda_{\ell}+\beta_{\ell}+y_{\ell}\right) .
\end{aligned}
$$

Substituting the values of parameters from (18) into (14) and using Eq. (15), we obtain

$$
\pm\left(\zeta-\zeta_{0}\right)=\chi \int \frac{d \Psi}{\sqrt{\Lambda(\Psi)}},
$$

where

$$
\begin{gathered}
\chi=\sqrt{\frac{\chi_{0}}{\eta_{4}}}, \\
\Lambda(\Psi)=\Psi^{4}+\frac{\eta_{3}}{\eta_{4}} \Psi^{3}+\frac{\eta_{2}}{\eta_{4}} \Psi^{2}+\frac{\eta_{1}}{\eta_{4}} \Psi+\frac{\eta_{0}}{\eta_{4}} .
\end{gathered}
$$


As a consequence, the following exact solutions can now be written for the coupled system (2).

For $\Lambda(\Psi)=\left(\Psi-v_{1}\right)^{4}$,

$$
\begin{aligned}
\mathfrak{p}(x, t) & =e^{i\left[-\kappa x+\frac{\eta_{1} \delta_{1}\left[a_{1}+3 c_{1} \kappa-b_{1} v-2 d_{1} \kappa v\right]-2 \kappa \delta_{0} \chi_{0}\left[a_{1} \kappa+c_{1} \kappa^{2}+\delta_{0}^{2}\left(\theta_{1}+\lambda_{1}+\beta_{1}+y_{1}\right)\right]}{\eta_{1} \delta_{1} d_{1}-2 \delta_{0} \chi_{0}\left(b_{1} \kappa+d_{1} \kappa^{2}-1\right)} t+\epsilon\right]} \\
\times & {\left[\delta_{0}+\delta_{1} v_{1} \pm \frac{\delta_{1} \chi}{x-v t-\zeta_{0}}\right], } \\
\mathfrak{q}(x, t)= & e^{i\left[-\kappa x+\frac{\eta_{1} \delta_{1}\left[a_{2}+3 c_{2} \kappa-b_{2} v-2 d_{2} \kappa v\right]-2 \kappa \delta_{0} \chi_{0}\left[a_{2} \kappa+c_{2} \kappa^{2}+\delta_{0}^{2}\left(\theta_{2}+\lambda_{2}+\beta_{2}+y_{2}\right)\right]}{\eta_{1} \delta_{1} d_{2}-2 \delta_{0} \chi_{0}\left(b_{2} \kappa+d_{2} \kappa^{2}-1\right)} t+\epsilon\right]} \\
\times & {\left[\delta_{0}+\delta_{1} v_{1} \pm \frac{\delta_{1} \chi}{x-v t-\zeta_{0}}\right] . }
\end{aligned}
$$

If $\Lambda(\Psi)=\left(\Psi-v_{1}\right)^{3}\left(\Psi-v_{2}\right)$ and $v_{2}>v_{1}$,

$$
\begin{aligned}
& \mathfrak{p}(x, t)=e^{i\left[-\kappa x+\frac{\eta_{1} \delta_{1}\left[a_{1}+3 c_{1} \kappa-b_{1} v-2 d_{1} \kappa v\right]-2 \kappa \delta_{0} \chi_{0}\left[a_{1} \kappa+c_{1} \kappa^{2}+\delta_{0}^{2}\left(\theta_{1}+\lambda_{1}+\beta_{1}+y_{1}\right)\right]}{\eta_{1} \delta_{1} d_{1}-2 \delta_{0} \chi_{0}\left(b_{1} \kappa+d_{1} \kappa^{2}-1\right)} t+\epsilon\right]} \\
& \times\left[\delta_{0}+\delta_{1} v_{1}+\frac{4 \delta_{1} \chi^{2}\left(v_{2}-v_{1}\right)}{4 \chi^{2}-\left[\left(v_{1}-v_{2}\right)\left(x-v t-\zeta_{0}\right)\right]^{2}}\right], \\
& \mathfrak{q}(x, t)=e^{i\left[-\kappa x+\frac{\eta_{1} \delta_{1}\left[a_{2}+3 c_{2} \kappa-b_{2} v-2 d_{2} \kappa v\right]-2 \kappa \delta_{0} \chi_{0}\left[a_{2} \kappa+c_{2} \kappa^{2}+\delta_{0}^{2}\left(\theta_{2}+\lambda_{2}+\beta_{2}+y_{2}\right)\right]}{\eta_{1} \delta_{1} d_{2}-2 \delta_{0} \chi_{0}\left(b_{2} \kappa+d_{2} \kappa^{2}-1\right)} t+\epsilon\right]} \\
& \times\left[\delta_{0}+\delta_{1} v_{1}+\frac{4 \delta_{1} \chi^{2}\left(v_{2}-v_{1}\right)}{4 \chi^{2}-\left[\left(v_{1}-v_{2}\right)\left(x-v t-\zeta_{0}\right)\right]^{2}}\right] \text {. }
\end{aligned}
$$

However, if $\Lambda(\Psi)=\left(\Psi-v_{1}\right)^{2}\left(\Psi-v_{2}\right)^{2}$,

$$
\begin{aligned}
\mathfrak{p}(x, t)= & e^{i\left[-\kappa x+\frac{\eta_{1} \delta_{1}\left[a_{1}+3 c_{1} \kappa-b_{1} v-2 d_{1} \kappa v\right]-2 \kappa \delta_{0} \chi_{0}\left[a_{1} \kappa+c_{1} \kappa^{2}+\delta_{0}^{2}\left(\theta_{1}+\lambda_{1}+\beta_{1}+y_{1}\right)\right]}{\eta_{1} \delta_{1} d_{1}-2 \delta_{0} \chi_{0}\left(b_{1} \kappa+d_{1} \kappa^{2}-1\right)} t+\epsilon\right]}, \\
\times & {\left[\delta_{0}+\delta_{1} v_{2}+\frac{\delta_{1}\left(v_{2}-v_{1}\right)}{\left.\exp \left\{\frac{v_{1}-v_{2}}{\chi}\left(x-v t-\zeta_{0}\right)\right\}-1\right]}\right] } \\
\mathfrak{q}(x, t)= & e^{i\left[-\kappa x+\frac{\eta_{1} \delta_{1}\left[a_{2}+3 c_{2} \kappa-b_{2} v-2 d_{2} \kappa v\right]-2 \kappa \delta_{0} \chi_{0}\left[a_{2} \kappa+c_{2} \kappa^{2}+\delta_{0}^{2}\left(\theta_{2}+\lambda_{2}+\beta_{2}+y_{2}\right)\right]}{\eta_{1} \delta_{1} d_{2}-2 \delta_{0} \chi_{0}\left(b_{2} \kappa+d_{2} \kappa^{2}-1\right)} t+\epsilon\right]} \\
\times & {\left[\delta_{0}+\delta_{1} v_{2}+\frac{\delta_{1}\left(v_{2}-v_{1}\right)}{\exp \left\{\frac{v_{1}-v_{2}}{\chi}\left(x-v t-\zeta_{0}\right)\right\}-1}\right] }
\end{aligned}
$$

and

$$
\begin{aligned}
\mathfrak{p}(x, t) & =e^{i\left[-\kappa x+\frac{\eta_{1} \delta_{1}\left[a_{1}+3 c_{1} \kappa-b_{1} v-2 d_{1} \kappa v\right]-2 \kappa \delta_{0} \chi_{0}\left[a_{1} \kappa+c_{1} \kappa^{2}+\delta_{0}^{2}\left(\theta_{1}+\lambda_{1}+\beta_{1}+y_{1}\right)\right]}{\eta_{1} \delta_{1} d_{1}-2 \delta_{0} \chi_{0}\left(b_{1} \kappa+d_{1} \kappa^{2}-1\right)} t+\epsilon\right]} \\
\times & {\left[\delta_{0}+\delta_{1} v_{1}+\frac{\delta_{1}\left(v_{1}-v_{2}\right)}{\exp \left\{\frac{v_{1}-v_{2}}{\chi}\left(x-v t-\zeta_{0}\right)\right\}-1}\right] }
\end{aligned}
$$




$$
\begin{aligned}
\mathfrak{q}(x, t) & =e^{i\left[-\kappa x+\frac{\eta_{1} \delta_{1}\left[a_{2}+3 c_{2} \kappa-b_{2} v-2 d_{2} \kappa v\right]-2 \kappa \delta_{0} \chi_{0}\left[a_{2} \kappa+c_{2} \kappa^{2}+\delta_{0}^{2}\left(\theta_{2}+\lambda_{2}+\beta_{2}+y_{2}\right)\right]}{\eta_{1} \delta_{1} d_{2}-2 \delta_{0} \chi_{0}\left(b_{2} \kappa+d_{2} \kappa^{2}-1\right)} t+\epsilon\right]} \\
\times & {\left[\delta_{0}+\delta_{1} v_{1}+\frac{\delta_{1}\left(v_{1}-v_{2}\right)}{\exp \left\{\frac{v_{1}-v_{2}}{\chi}\left(x-v t-\zeta_{0}\right)\right\}-1}\right] }
\end{aligned}
$$

Whenever $\Lambda(\Psi)=\left(\Psi-v_{1}\right)^{2}\left(\Psi-v_{2}\right)\left(\Psi-v_{3}\right)$ and $v_{1}>v_{2}>v_{3}$,

$$
\begin{aligned}
& \mathfrak{p}(x, t)=e^{i\left[-\kappa x+\frac{\eta_{1} \delta_{1}\left[a_{1}+3 c_{1} \kappa-b_{1} v-2 d_{1} \kappa v\right]-2 \kappa \delta_{0} \chi_{0}\left[a_{1} \kappa+c_{1} \kappa^{2}+\delta_{0}^{2}\left(\theta_{1}+\lambda_{1}+\beta_{1}+y_{1}\right)\right]}{\eta_{1} \delta_{1} d_{1}-2 \delta_{0} \chi_{0}\left(b_{1} \kappa+d_{1} \kappa^{2}-1\right)} t+\epsilon\right]} \\
& \left.\times\left[\delta_{0}+\delta_{1} v_{1}-\frac{2 \delta_{1}\left(v_{1}-v_{2}\right)\left(v_{1}-v_{3}\right)}{2 v_{1}-v_{2}-v_{3}+\left(v_{3}-v_{2}\right) \cosh \left(\frac{\sqrt{\left(v_{1}-v_{2}\right)\left(v_{1}-v_{3}\right)}}{\chi}\left(x-v t-\zeta_{0}\right)\right.}\right)\right], \\
& \mathfrak{q}(x, t)=e^{i\left[-\kappa x+\frac{\eta_{1} \delta_{1}\left[a_{2}+3 c_{2} \kappa-b_{2} v-2 d_{2} \kappa v\right]-2 \kappa \delta_{0} \chi_{0}\left[a_{2} \kappa+c_{2} \kappa^{2}+\delta_{0}^{2}\left(\theta_{2}+\lambda_{2}+\beta_{2}+y_{2}\right)\right]}{\eta_{1} \delta_{1} d_{2}-2 \delta_{0} \chi_{0}\left(b_{2} \kappa+d_{2} \kappa^{2}-1\right)} t+\epsilon\right]} \\
& \left.\times\left[\delta_{0}+\delta_{1} v_{1}-\frac{2 \delta_{1}\left(v_{1}-v_{2}\right)\left(v_{1}-v_{3}\right)}{2 v_{1}-v_{2}-v_{3}+\left(v_{3}-v_{2}\right) \cosh \left(\frac{\sqrt{\left(v_{1}-v_{2}\right)\left(v_{1}-v_{3}\right)}}{\chi}\left(x-v t-\zeta_{0}\right)\right.}\right)\right] .
\end{aligned}
$$

Finally, if $\Lambda(\Psi)=\left(\Psi-v_{1}\right)\left(\Psi-v_{2}\right)\left(\Psi-v_{3}\right)\left(\Psi-v_{4}\right)$ and $v_{1}>v_{2}>v_{3}>v_{4}$,

$$
\begin{aligned}
\mathfrak{p}(x, t) & \left.=e^{i\left[-\kappa x+\frac{\eta_{1} \delta_{1}\left[a_{1}+3 c_{1} \kappa-b_{1} v-2 d_{1} \kappa v\right]-2 \kappa \delta_{0} \chi_{0}\left[a_{1} \kappa+c_{1} \kappa^{2}+\delta_{0}^{2}\left(\theta_{1}+\lambda_{1}+\beta_{1}+y_{1}\right)\right]}{\eta_{1} \delta_{1} d_{1}-2 \delta_{0} \chi_{0}\left(b_{1} \kappa+d_{1} \kappa^{2}-1\right)} t+\epsilon\right]}\right] \\
\times & {\left[\delta_{0}+\delta_{1} v_{2}+\frac{\delta_{1}\left(v_{1}-v_{2}\right)\left(v_{4}-v_{2}\right)}{v_{4}-v_{2}+\left(v_{1}-v_{4}\right) s n^{2}\left( \pm \frac{\sqrt{\left(v_{1}-v_{3}\right)\left(v_{2}-v_{4}\right)}}{2 \chi}\left(x-v t-\zeta_{0}\right), m\right)}\right], } \\
\mathfrak{q}(x, t)= & \left.e^{i\left[-\kappa x+\frac{\eta_{1} \delta_{1}\left[a_{2}+3 c_{2} \kappa-b_{2} v-2 d_{2} \kappa v\right]-2 \kappa \delta_{0} \chi_{0}\left[a_{2} \kappa+c_{2} \kappa^{2}+\delta_{0}^{2}\left(\theta_{2}+\lambda_{2}+\beta_{2}+y_{2}\right)\right]}{\eta_{1} \delta_{1} d_{2}-2 \delta_{0} \chi_{0}\left(b_{2} \kappa+d_{2} \kappa^{2}-1\right)} t+\epsilon\right]}\right] \\
\times & {\left[\delta_{0}+\delta_{1} v_{2}+\frac{\delta_{1}\left(v_{1}-v_{2}\right)\left(v_{4}-v_{2}\right)}{v_{4}-v_{2}+\left(v_{1}-v_{4}\right) s n^{2}\left( \pm \frac{\sqrt{\left(v_{1}-v_{3}\right)\left(v_{2}-v_{4}\right)}}{2 \chi}\left(x-v t-\zeta_{0}\right), m\right)}\right], }
\end{aligned}
$$

where

$$
m^{2}=\frac{\left(v_{2}-v_{3}\right)\left(v_{1}-v_{4}\right)}{\left(v_{1}-v_{3}\right)\left(v_{2}-v_{4}\right)},
$$

and $v_{j}, j=1, \ldots, 4$ are the roots of $\Lambda(\Psi)=0$.

By assuming $\delta_{0}=-\delta_{1} v_{1}$ and $\zeta_{0}=0$, the solutions given by (23)-(32) can be transformed to the plane wave solutions

$$
\begin{aligned}
\mathfrak{p}(x, t) & =e^{i\left[-\kappa x+\frac{\eta_{1} \delta_{1}\left[a_{1}+3 c_{1} \kappa-b_{1} v-2 d_{1} \kappa v\right]-2 \kappa \delta_{0} \chi_{0}\left[a_{1} \kappa+c_{1} \kappa^{2}+\delta_{0}^{2}\left(\theta_{1}+\lambda_{1}+\beta_{1}+y_{1}\right)\right]}{\eta_{1} \delta_{1} d_{1}-2 \delta_{0} \chi_{0}\left(b_{1} \kappa+d_{1} \kappa^{2}-1\right)} t+\epsilon\right]} \\
& \times\left( \pm \frac{\delta_{1} \chi}{x-v t}\right)
\end{aligned}
$$




$$
\begin{aligned}
& \mathfrak{q}(x, t)=e^{i\left[-\kappa x+\frac{\eta_{1} \delta_{1}\left[a_{2}+3 c_{2} \kappa-b_{2} v-2 d_{2} \kappa v\right]-2 \kappa \delta_{0} \chi_{0}\left[a_{2} \kappa+c_{2} \kappa^{2}+\delta_{0}^{2}\left(\theta_{2}+\lambda_{2}+\beta_{2}+y_{2}\right)\right]}{\eta_{1} \delta_{1} d_{2}-2 \delta_{0} \chi_{0}\left(b_{2} \kappa+d_{2} \kappa^{2}-1\right)} t+\epsilon\right]} \\
& \times\left( \pm \frac{\delta_{1} \chi}{x-v t}\right), \\
& \mathfrak{p}(x, t)=e^{i\left[-\kappa x+\frac{\eta_{1} \delta_{1}\left[a_{1}+3 c_{1} \kappa-b_{1} v-2 d_{1} \kappa v\right]-2 \kappa \delta_{0} \chi_{0}\left[a_{1} \kappa+c_{1} \kappa^{2}+\delta_{0}^{2}\left(\theta_{1}+\lambda_{1}+\beta_{1}+y_{1}\right)\right]}{\eta_{1} \delta_{1} d_{1}-2 \delta_{0} \chi_{0}\left(b_{1} \kappa+d_{1} \kappa^{2}-1\right)} t+\epsilon\right]} \\
& \times\left(\frac{4 \delta_{1} \chi^{2}\left(v_{2}-v_{1}\right)}{4 \chi^{2}-\left[\left(v_{1}-v_{2}\right)(x-v t)\right]^{2}}\right), \\
& \mathfrak{q}(x, t)=e^{i\left[-\kappa x+\frac{\eta_{1} \delta_{1}\left[a_{2}+3 c_{2} \kappa-b_{2} v-2 d_{2} \kappa v\right]-2 \kappa \delta_{0} \chi_{0}\left[a_{2} \kappa+c_{2} \kappa^{2}+\delta_{0}^{2}\left(\theta_{2}+\lambda_{2}+\beta_{2}+y_{2}\right)\right]}{\eta_{1} \delta_{1} d_{2}-2 \delta_{0} \chi_{0}\left(b_{2} \kappa+d_{2} \kappa^{2}-1\right)} t+\epsilon\right]} \\
& \times\left(\frac{4 \delta_{1} \chi^{2}\left(v_{2}-v_{1}\right)}{4 \chi^{2}-\left[\left(v_{1}-v_{2}\right)(x-v t)\right]^{2}}\right),
\end{aligned}
$$

singular soliton solutions

$$
\begin{aligned}
\mathfrak{p}(x, t)= & e^{i\left[-\kappa x+\frac{\eta_{1} \delta_{1}\left[a_{1}+3 c_{1} \kappa-b_{1} v-2 d_{1} \kappa v\right]-2 \kappa \delta_{0} \chi_{0}\left[a_{1} \kappa+c_{1} \kappa^{2}+\delta_{0}^{2}\left(\theta_{1}+\lambda_{1}+\beta_{1}+y_{1}\right)\right]}{\eta_{1} \delta_{1} d_{1}-2 \delta_{0} \chi_{0}\left(b_{1} \kappa+d_{1} \kappa^{2}-1\right)} t+\epsilon\right]} \\
\times & {\left[\frac{\delta_{1}\left(v_{2}-v_{1}\right)}{2}\left\{1 \mp \operatorname{coth}\left(\frac{v_{1}-v_{2}}{2 \chi}(x-v t)\right)\right\}\right], } \\
\mathfrak{q}(x, t)= & e^{i\left[-\kappa x+\frac{\eta_{1} \delta_{1}\left[a_{2}+3 c_{2} \kappa-b_{2} v-2 d_{2} \kappa v\right]-2 \kappa \delta_{0} \chi_{0}\left[a_{2} \kappa+c_{2} \kappa^{2}+\delta_{0}^{2}\left(\theta_{2}+\lambda_{2}+\beta_{2}+y_{2}\right)\right]}{\eta_{1} \delta_{1} d_{2}-2 \delta_{0} \chi_{0}\left(b_{2} \kappa+d_{2} \kappa^{2}-1\right)} t+\epsilon\right]} \\
\times & {\left[\frac{\delta_{1}\left(v_{2}-v_{1}\right)}{2}\left\{1 \mp \operatorname{coth}\left(\frac{v_{1}-v_{2}}{2 \chi}(x-v t)\right)\right\}\right], }
\end{aligned}
$$

bright soliton solutions

$$
\begin{aligned}
\mathfrak{p}(x, t)= & \left.e^{i\left[-\kappa x+\frac{\eta_{1} \delta_{1}\left[a_{1}+3 c_{1} \kappa-b_{1} v-2 d_{1} \kappa v\right]-2 \kappa \delta_{0} \chi_{0}\left[a_{1} \kappa+c_{1} \kappa^{2}+\delta_{0}^{2}\left(\theta_{1}+\lambda_{1}+\beta_{1}+y_{1}\right)\right]}{\eta_{1} \delta_{1} d_{1}-2 \delta_{0} \chi_{0}\left(b_{1} \kappa+d_{1} \kappa^{2}-1\right)} t+\epsilon\right]}\right] \\
\times & {\left[\frac{-2 \delta_{1}\left(v_{1}-v_{2}\right)\left(v_{1}-v_{3}\right)}{2 v_{1}-v_{2}-v_{3}+\left(v_{3}-v_{2}\right) \cosh \left(\frac{\sqrt{\left(v_{1}-v_{2}\right)\left(v_{1}-v_{3}\right)}}{\chi}(x-v t)\right)}\right] } \\
\mathfrak{q}(x, t)= & \left.e^{i\left[-\kappa x+\frac{\eta_{1} \delta_{1}\left[a_{2}+3 c_{2} \kappa-b_{2} v-2 d_{2} \kappa v\right]-2 \kappa \delta_{0} \chi_{0}\left[a_{2} \kappa+c_{2} \kappa^{2}+\delta_{0}^{2}\left(\theta_{2}+\lambda_{2}+\beta_{2}+y_{2}\right)\right]}{\eta_{1} \delta_{1} d_{2}-2 \delta_{0} \chi_{0}\left(b_{2} \kappa+d_{2} \kappa^{2}-1\right)} t+\epsilon\right]}\right] \\
\times & {\left[\frac{-2 \delta_{1}\left(v_{1}-v_{2}\right)\left(v_{1}-v_{3}\right)}{\chi v_{1}-v_{2}-v_{3}+\left(v_{3}-v_{2}\right) \cosh \left(\frac{\sqrt{\left(v_{1}-v_{2}\right)\left(v_{1}-v_{3}\right)}}{\chi}(x-v t)\right)}\right] }
\end{aligned}
$$

Moreover, when $\delta_{0}=-\delta_{1} \nu_{2}$ and $\zeta_{0}=0$, Jacobi elliptic function solutions (33) and (34) trimmed as

$$
\begin{aligned}
\mathfrak{p}(x, t) & =e^{i\left[-\kappa x+\frac{\eta_{1} \delta_{1}\left[a_{1}+3 c_{1} \kappa-b_{1} v-2 d_{1} \kappa v\right]-2 \kappa \delta_{0} \chi_{0}\left[a_{1} \kappa+c_{1} \kappa^{2}+\delta_{0}^{2}\left(\theta_{1}+\lambda_{1}+\beta_{1}+y_{1}\right)\right]}{\eta_{1} \delta_{1} d_{1}-2 \delta_{0} \chi_{0}\left(b_{1} \kappa+d_{1} \kappa^{2}-1\right)} t+\epsilon\right]} \\
& \times\left[\frac{\delta_{1}\left(v_{1}-v_{2}\right)\left(v_{4}-v_{2}\right)}{v_{4}-v_{2}+\left(v_{1}-v_{4}\right) \operatorname{sn}^{2}\left( \pm \frac{\sqrt{\left(v_{1}-v_{3}\right)\left(v_{2}-v_{4}\right)}}{2 \chi}(x-v t), \frac{\left(v_{2}-v_{3}\right)\left(v_{1}-v_{4}\right)}{\left(v_{1}-v_{3}\right)\left(v_{2}-v_{4}\right)}\right)}\right],
\end{aligned}
$$




$$
\begin{aligned}
& \mathfrak{q}(x, t)=e^{i\left[-\kappa x+\frac{\eta_{1} \delta_{1}\left[a_{2}+3 c_{2} \kappa-b_{2} v-2 d_{2} \kappa v\right]-2 \kappa \delta_{0} \chi_{0}\left[a_{2} \kappa+c_{2} \kappa^{2}+\delta_{0}^{2}\left(\theta_{2}+\lambda_{2}+\beta_{2}+y_{2}\right)\right]}{\eta_{1} \delta_{1} d_{2}-2 \delta_{0} \chi_{0}\left(b_{2} \kappa+d_{2} \kappa^{2}-1\right)} t+\epsilon\right]} \\
& \times\left[\frac{\delta_{1}\left(v_{1}-v_{2}\right)\left(v_{4}-v_{2}\right)}{v_{4}-v_{2}+\left(v_{1}-v_{4}\right) \operatorname{sn}^{2}\left( \pm \frac{\sqrt{\left(v_{1}-v_{3}\right)\left(v_{2}-v_{4}\right)}}{2 \chi}(x-v t), \frac{\left(v_{2}-v_{3}\right)\left(v_{1}-v_{4}\right)}{\left(v_{1}-v_{3}\right)\left(v_{2}-v_{4}\right)}\right)}\right] \text {. }
\end{aligned}
$$

Remark 1. When the modulus $m \rightarrow 1$, singular soliton solution emerge as

$$
\begin{aligned}
& \mathfrak{p}(x, t)=e^{i\left[-\kappa x+\frac{\eta_{1} \delta_{1}\left[a_{1}+3 c_{1} \kappa-b_{1} v-2 d_{1} \kappa v\right]-2 \kappa \delta_{0} \chi_{0}\left[a_{1} \kappa+c_{1} \kappa^{2}+\delta_{0}^{2}\left(\theta_{1}+\lambda_{1}+\beta_{1}+y_{1}\right)\right]}{\eta_{1} \delta_{1} d_{1}-2 \delta_{0} \chi_{0}\left(b_{1} \kappa+d_{1} \kappa^{2}-1\right)} t+\epsilon\right]} \\
& \times\left[\frac{\delta_{1}\left(v_{1}-v_{2}\right)\left(v_{4}-v_{2}\right)}{v_{4}-v_{2}+\left(v_{1}-v_{4}\right) \tanh ^{2}\left( \pm \frac{\sqrt{\left(v_{1}-v_{3}\right)\left(v_{2}-v_{4}\right)}}{2 \chi}(x-v t)\right)}\right], \\
& \mathfrak{q}(x, t)=e^{i\left[-\kappa x+\frac{\eta_{1} \delta_{1}\left[a_{2}+3 c_{2} \kappa-b_{2} v-2 d_{2} \kappa v\right]-2 \kappa \delta_{0} \chi_{0}\left[a_{2} \kappa+c_{2} \kappa^{2}+\delta_{0}^{2}\left(\theta_{2}+\lambda_{2}+\beta_{2}+y_{2}\right)\right]}{\eta_{1} \delta_{1} d_{2}-2 \delta_{0} \chi_{0}\left(b_{2} \kappa+d_{2} \kappa^{2}-1\right)} t+\epsilon\right]} \\
& \times\left[\frac{\delta_{1}\left(v_{1}-v_{2}\right)\left(v_{4}-v_{2}\right)}{v_{4}-v_{2}+\left(v_{1}-v_{4}\right) \tanh ^{2}\left( \pm \frac{\sqrt{\left(v_{1}-v_{3}\right)\left(v_{2}-v_{4}\right)}}{2 \chi}(x-v t)\right)}\right],
\end{aligned}
$$

where $v_{3}=v_{4}$.

Remark 2. When the modulus $m \rightarrow 0$, periodic singular solution emerge as

$$
\begin{aligned}
\mathfrak{p}(x, t) & \left.=e^{i\left[-\kappa x+\frac{\eta_{1} \delta_{1}\left[a_{1}+3 c_{1} \kappa-b_{1} v-2 d_{1} \kappa v\right]-2 \kappa \delta_{0} \chi_{0}\left[a_{1} \kappa+c_{1} \kappa^{2}+\delta_{0}^{2}\left(\theta_{1}+\lambda_{1}+\beta_{1}+y_{1}\right)\right]}{\eta_{1} \delta_{1} d_{1}-2 \delta_{0} \chi_{0}\left(b_{1} \kappa+d_{1} \kappa^{2}-1\right)} t+\epsilon\right]}\right], \\
\times & {\left[\frac{\delta_{1}\left(v_{1}-v_{2}\right)\left(v_{4}-v_{2}\right)}{v_{4}-v_{2}+\left(v_{1}-v_{4}\right) \sin ^{2}\left( \pm \frac{\sqrt{\left(v_{1}-v_{3}\right)\left(v_{2}-v_{4}\right)}}{2 \chi}(x-v t)\right)}\right] } \\
\mathfrak{q}(x, t)= & \left.e^{i\left[-\kappa x+\frac{\eta_{1} \delta_{1}\left[a_{2}+3 c_{2} \kappa-b_{2} v-2 d_{2} \kappa v\right]-2 \kappa \delta_{0} \chi_{0}\left[a_{2} \kappa+c_{2} \kappa^{2}+\delta_{0}^{2}\left(\theta_{2}+\lambda_{2}+\beta_{2}+y_{2}\right)\right]}{\eta_{1} \delta_{1} d_{2}-2 \delta_{0} \chi_{0}\left(b_{2} \kappa+d_{2} \kappa^{2}-1\right)} t+\epsilon\right]}\right] \\
\times & {\left[\frac{\delta_{1}\left(v_{1}-v_{2}\right)\left(v_{4}-v_{2}\right)}{2 \chi}\right] }
\end{aligned}
$$

where $v_{2}=v_{3}$.

Case-2. Eq. (12) can be written as

$$
\begin{array}{r}
\left(a_{\ell}-v b_{\ell}-2 \kappa v d_{\ell}+3 \kappa c_{\ell}-\omega d_{\ell}\right)\left\{2 P_{\ell} P_{\ell}^{\prime \prime}-\left(P_{\ell}^{\prime}\right)^{2}\right\}-4 \kappa\left(\theta_{\ell}+\lambda_{\ell}+\beta_{\ell}+y_{\ell}\right) P_{\ell}^{3} \\
-4\left(\omega+a_{\ell} \kappa^{2}+c_{\ell} \kappa^{3}-b_{\ell} \omega \kappa-d_{\ell} \kappa^{2} \omega\right) P_{\ell}^{2}=0,
\end{array}
$$

through the transformation $\mathfrak{Q}_{\ell}=P_{\ell}^{1 / 2}$. Therefore, the solution of Eq. (50) can be expressed as

$$
P_{\ell}=\sum_{i=0}^{\varrho} \delta_{i} \Psi^{i}
$$


where $\delta_{i}$ are unknown constant to be determined such that $\delta_{\varrho} \neq 0$.

The balancing process reveals that

$$
\sigma=\rho+\varrho+2
$$

By assuming $\sigma=3, \rho=0$ and $\varrho=1$ in Eq. (52), we arrive at

$$
P_{\ell}=\delta_{0}+\delta_{1} \Psi .
$$

Implanting Eq. (53) along with Eq. (14) into Eq. (50) and evaluating the resultant system of equations, we attain

$$
\begin{gathered}
\chi_{0}=\chi_{0}, \quad \delta_{0}=\delta_{0}, \quad \delta_{1}=\delta_{1}, \quad \eta_{3}=\eta_{3}, \\
\omega=\frac{2 \eta_{3}\left(a_{\ell}+3 c_{\ell} \kappa-b_{\ell} v-2 d_{\ell} \kappa v\right)-4 \kappa \delta_{1} \chi_{0}\left(\theta_{\ell}+\lambda_{\ell}+\beta_{\ell}+y_{\ell}\right)}{2 \eta_{3} d_{\ell}}, \\
\eta_{0}=\frac{\delta_{0}^{2}\left[k_{1}-2 a_{\ell} \eta_{3}\left(b_{\ell} \kappa-1\right)+4 \delta_{1} \chi_{0} \kappa\left(\theta_{\ell}+\lambda_{\ell}+\beta_{\ell}+y_{\ell}\right)\left(b_{\ell} \kappa+d_{\ell} \kappa^{2}-1\right)\right]}{d_{\ell} \delta_{1}^{3} \kappa\left(\theta_{\ell}+\lambda_{\ell}+\beta_{\ell}+y_{\ell}\right)}, \\
\eta_{1}=\frac{\delta_{0}\left[k_{2}+k_{3}+4 b_{\ell}^{2} v \kappa \eta_{3}-4 a_{\ell} \eta_{3}\left(b_{\ell} \kappa-1\right)+8 \delta_{1} \chi_{0} \kappa\left(d_{\ell} \kappa^{2}-1\right)\left(\theta_{\ell}+\lambda_{\ell}+\beta_{\ell}+y_{\ell}\right)\right]}{d_{\ell} \delta_{1}^{2} \kappa\left(\theta_{\ell}+\lambda_{\ell}+\beta_{\ell}+y_{\ell}\right)}, \\
\eta_{2}=\frac{k_{4}-2 a_{\ell} \eta_{3}\left(b_{\ell} \kappa-1\right)+4 \kappa \delta_{1} \chi_{0}\left(\theta_{\ell}+\lambda_{\ell}+\beta_{\ell}+y_{\ell}\right)\left(b_{\ell} \kappa+d_{\ell} \kappa^{2}-1\right)}{d_{\ell} \delta_{1} \kappa\left(\theta_{\ell}+\lambda_{\ell}+\beta_{\ell}+y_{\ell}\right)}
\end{gathered}
$$

where

$$
\begin{gathered}
k_{1}=2 \eta_{3}\left[d_{\ell} \kappa\left\{3 b_{\ell} v \kappa-2\left(c_{\ell} \kappa^{2}+v\right)\right\}+\left(b_{\ell} \kappa-1\right)\left(b_{\ell} v-3 c_{\ell} \kappa\right)+2 d_{\ell}^{2} v \kappa^{3}\right]+\eta_{3} d_{\ell} \kappa \delta_{0}\left(\theta_{\ell}+\lambda_{\ell}+\beta_{\ell}+y_{\ell}\right), \\
k_{2}=3 d_{\ell} \delta_{0} \kappa \eta_{3}\left(\theta_{\ell}+\lambda_{\ell}+\beta_{\ell}+y_{\ell}\right)+4 \kappa \eta_{3}\left\{2 d_{\ell}^{2} v \kappa^{2}-2 d_{\ell}\left(c_{\ell} \kappa^{2}+v\right)+3 c_{\ell} \kappa \eta_{3}\right\}, \\
k_{3}=-4 b_{\ell} \eta_{3}\left\{3 \kappa^{2}\left(c_{\ell}-d_{\ell} v\right)+v\right\}+8 b_{\ell} \kappa^{2} \delta_{1} \chi_{0}\left(\theta_{\ell}+\lambda_{\ell}+\beta_{\ell}+y_{\ell}\right), \\
k_{4}=2 \eta_{3}\left[d_{\ell} \kappa\left\{3 b_{\ell} v \kappa-2\left(c_{\ell} \kappa^{2}+v\right)\right\}+\left(b_{\ell} \kappa-1\right)\left(b_{\ell} v-3 c_{\ell} \kappa\right)+2 d_{\ell}^{2} v \kappa^{3}\right]+3 \eta_{3} d_{\ell} \kappa \delta_{0}\left(\theta_{\ell}+\lambda_{\ell}+\beta_{\ell}+y_{\ell}\right) .
\end{gathered}
$$

Substituting all the values from (54) into (14) and using Eq. (15), we obtain

$$
\pm\left(\zeta-\zeta_{0}\right)=\chi \int \frac{d \Psi}{\sqrt{\Lambda(\Psi)}},
$$

where

$$
\begin{gathered}
\chi=\sqrt{\frac{\chi_{0}}{\eta_{3}}}, \\
\Lambda(\Psi)=\Psi^{3}+\frac{\eta_{2}}{\eta_{3}} \Psi^{2}+\frac{\eta_{1}}{\eta_{3}} \Psi+\frac{\eta_{0}}{\eta_{3}} .
\end{gathered}
$$

As a consequence, the following exact solutions can now be written for the coupled system (2).

For $\Lambda(\Psi)=\left(\Psi-v_{1}\right)^{3}$,

$$
\begin{aligned}
\mathfrak{p}(x, t) & =e^{i\left[-\kappa x+\frac{2 \eta_{3}\left(a_{1}+3 c_{1} \kappa-b_{1} v-2 d_{1} \kappa v\right)-4 \kappa \delta_{1} \chi_{0}\left(\theta_{1}+\lambda_{1}+\beta_{1}+y_{1}\right)}{2 \eta_{3} d_{1}} t+\epsilon\right]} \\
\times & {\left[\delta_{0}+\delta_{1} v_{1}+\frac{4 \delta_{1} \chi}{\left(x-v t-\zeta_{0}\right)^{2}}\right]^{1 / 2}, } \\
\mathfrak{q}(x, t)= & e^{i\left[-\kappa x+\frac{2 \eta_{3}\left(a_{2}+3 c_{2} \kappa-b_{2} v-2 d_{2} \kappa v\right)-4 \kappa \delta_{1} \chi_{0}\left(\theta_{2}+\lambda_{2}+\beta_{2}+y_{2}\right)}{2 \eta_{3} d_{2}} t+\epsilon\right]} \\
\times & {\left[\delta_{0}+\delta_{1} v_{1}+\frac{4 \delta_{1} \chi}{\left(x-v t-\zeta_{0}\right)^{2}}\right]^{1 / 2} . }
\end{aligned}
$$


If $\Lambda(\Psi)=\left(\Psi-v_{1}\right)^{2}\left(\Psi-v_{2}\right)$ and $v_{2}>v_{1}$,

$$
\begin{aligned}
& \mathfrak{p}(x, t)=e^{i\left[-\kappa x+\frac{2 \eta_{3}\left(a_{1}+3 c_{1} \kappa-b_{1} v-2 d_{1} \kappa v\right)-4 \kappa \delta_{1} \chi_{0}\left(\theta_{1}+\lambda_{1}+\beta_{1}+y_{1}\right)}{2 \eta_{3} d_{1}} t+\epsilon\right]} \\
& \times\left[\delta_{0}+\delta_{1} v_{2}+\delta_{1}\left(v_{1}-v_{2}\right) \tanh ^{2}\left(\frac{1}{2} \sqrt{\frac{v_{1}-v_{2}}{\chi}}\left(x-v t-\zeta_{0}\right)\right)\right]^{1 / 2}, \\
& \mathfrak{q}(x, t)=e^{i\left[-\kappa x+\frac{2 \eta_{3}\left(a_{2}+3 c_{2} \kappa-b_{2} v-2 d_{2} \kappa v\right)-4 \kappa \delta_{1} \chi_{0}\left(\theta_{2}+\lambda_{2}+\beta_{2}+y_{2}\right)}{2 \eta_{3} d_{2}} t+\epsilon\right]} \\
& \times\left[\delta_{0}+\delta_{1} v_{2}+\delta_{1}\left(v_{1}-v_{2}\right) \tanh ^{2}\left(\frac{1}{2} \sqrt{\frac{v_{1}-v_{2}}{\chi}}\left(x-v t-\zeta_{0}\right)\right)\right]^{1 / 2} .
\end{aligned}
$$

However, if $\Lambda(\Psi)=\left(\Psi-v_{1}\right)\left(\Psi-v_{2}\right)^{2}$, and $v_{1}>v_{2}$,

$$
\begin{aligned}
\mathfrak{p}(x, t)= & e^{i\left[-\kappa x+\frac{2 \eta_{3}\left(a_{1}+3 c_{1} \kappa-b_{1} v-2 d_{1} \kappa v\right)-4 \kappa \delta_{1} \chi_{0}\left(\theta_{1}+\lambda_{1}+\beta_{1}+y_{1}\right)}{2 \eta_{3} d_{1}} t+\epsilon\right]} \\
\times & {\left[\delta_{0}+\delta_{1} v_{1}+\delta_{1}\left(v_{1}-v_{2}\right) \operatorname{csch}^{2}\left(\frac{1}{2} \sqrt{\frac{v_{1}-v_{2}}{\chi}}\left(x-v t-\zeta_{0}\right)\right)\right]^{1 / 2}, } \\
\mathfrak{q}(x, t)= & e^{i\left[-\kappa x+\frac{2 \eta_{3}\left(a_{2}+3 c_{2} \kappa-b_{2} v-2 d_{2} \kappa v\right)-4 \kappa \delta_{1} \chi_{0}\left(\theta_{2}+\lambda_{2}+\beta_{2}+y_{2}\right)}{2 \eta_{3} d_{2}} t+\epsilon\right]} \\
\times & {\left[\delta_{0}+\delta_{1} v_{1}+\delta_{1}\left(v_{1}-v_{2}\right) \operatorname{csch}^{2}\left(\frac{1}{2} \sqrt{\frac{v_{1}-v_{2}}{\chi}}\left(x-v t-\zeta_{0}\right)\right)\right]^{1 / 2} . }
\end{aligned}
$$

Whenever $\Lambda(\Psi)=\left(\Psi-v_{1}\right)\left(\Psi-v_{2}\right)\left(\Psi-v_{3}\right)$ and $v_{1}>v_{2}>v_{3}$,

$$
\begin{aligned}
\mathfrak{p}(x, t)= & e^{i\left[-\kappa x+\frac{2 \eta_{3}\left(a_{1}+3 c_{1} \kappa-b_{1} v-2 d_{1} \kappa v\right)-4 \kappa \delta_{1} \chi_{0}\left(\theta_{1}+\lambda_{1}+\beta_{1}+y_{1}\right)}{2 \eta_{3} d_{1}} t+\epsilon\right]} \\
\times & {\left[\delta_{0}+\delta_{1} v_{3}+\delta_{1}\left(v_{2}-v_{3}\right) \operatorname{sn}^{2}\left(\mp \frac{1}{2} \sqrt{\frac{v_{1}-v_{3}}{\chi}}\left(x-v t-\zeta_{0}\right), m\right)\right]^{1 / 2}, } \\
\mathfrak{q}(x, t)= & e^{i\left[-\kappa x+\frac{2 \eta_{3}\left(a_{2}+3 c_{2} \kappa-b_{2} v-2 d_{2} \kappa v\right)-4 \kappa \delta_{1} \chi_{0}\left(\theta_{2}+\lambda_{2}+\beta_{2}+y_{2}\right)}{2 \eta_{3} d_{2}} t+\epsilon\right]} \\
\times & {\left[\delta_{0}+\delta_{1} v_{3}+\delta_{1}\left(v_{2}-v_{3}\right) \operatorname{sn}^{2}\left(\mp \frac{1}{2} \sqrt{\frac{v_{1}-v_{3}}{\chi}}\left(x-v t-\zeta_{0}\right), m\right)\right]^{1 / 2}, }
\end{aligned}
$$

where

$$
m^{2}=\frac{\left(v_{2}-v_{3}\right)}{\left(v_{1}-v_{3}\right)}
$$

and $v_{j}, j=1,2,3$ are the roots of $\Lambda(\Psi)=0$.

By assuming $\delta_{0}=-\delta_{1} v_{1}$ and $\zeta_{0}=0$, the solutions given by (59)-(64) can be transformed to rational solutions

$$
\begin{aligned}
& \mathfrak{p}(x, t)=\frac{\sqrt{4 \delta_{1} \chi}}{x-v t} \cdot e^{i\left[-\kappa x+\frac{2 \eta_{3}\left(a_{1}+3 c_{1} \kappa-b_{1} v-2 d_{1} \kappa v\right)-4 \kappa \delta_{1} \chi_{0}\left(\theta_{1}+\lambda_{1}+\beta_{1}+y_{1}\right)}{2 \eta_{3} d_{1}} t+\epsilon\right],} \\
& \mathfrak{q}(x, t)=\frac{\sqrt{4 \delta_{1} \chi}}{x-v t} \cdot e^{i\left[-\kappa x+\frac{2 \eta_{3}\left(a_{2}+3 c_{2} \kappa-b_{2} v-2 d_{2} \kappa v\right)-4 \kappa \delta_{1} \chi_{0}\left(\theta_{2}+\lambda_{2}+\beta_{2}+y_{2}\right)}{2 \eta_{3} d_{2}} t+\epsilon\right]},
\end{aligned}
$$

bright soliton solutions

$$
\begin{aligned}
\mathfrak{p}(x, t) & =e^{i\left[-\kappa x+\frac{2 \eta_{3}\left(a_{1}+3 c_{1} \kappa-b_{1} v-2 d_{1} \kappa v\right)-4 \kappa \delta_{1} \chi_{0}\left(\theta_{1}+\lambda_{1}+\beta_{1}+y_{1}\right)}{2 \eta_{3} d_{1}} t+\epsilon\right]} \\
& \times\left[\sqrt{\delta_{1}\left(v_{2}-v_{1}\right)} \operatorname{sech}\left(\frac{1}{2} \sqrt{\frac{v_{1}-v_{2}}{\chi}}(x-v t)\right)\right]
\end{aligned}
$$




$$
\begin{aligned}
\mathfrak{q}(x, t) & =e^{i\left[-\kappa x+\frac{2 \eta_{3}\left(a_{2}+3 c_{2} \kappa-b_{2} v-2 d_{2} \kappa v\right)-4 \kappa \delta_{1} \chi_{0}\left(\theta_{2}+\lambda_{2}+\beta_{2}+y_{2}\right)}{2 \eta_{3} d_{2}} t+\epsilon\right]} \\
& \times\left[\sqrt{\delta_{1}\left(v_{2}-v_{1}\right)} \operatorname{sech}\left(\frac{1}{2} \sqrt{\frac{v_{1}-v_{2}}{\chi}}(x-v t)\right)\right]
\end{aligned}
$$

and singular soliton solutions

$$
\begin{aligned}
\mathfrak{p}(x, t) & =e^{i\left[-\kappa x+\frac{2 \eta_{3}\left(a_{1}+3 c_{1} \kappa-b_{1} v-2 d_{1} \kappa v\right)-4 \kappa \delta_{1} \chi_{0}\left(\theta_{1}+\lambda_{1}+\beta_{1}+y_{1}\right)}{2 \eta_{3} d_{1}} t+\epsilon\right]} \\
\times & {\left[\sqrt{\delta_{1}\left(v_{1}-v_{2}\right)} \operatorname{csch}\left(\frac{1}{2} \sqrt{\frac{v_{1}-v_{2}}{\chi}}(x-v t)\right)\right], } \\
\mathfrak{q}(x, t)= & e^{i\left[-\kappa x+\frac{2 \eta_{3}\left(a_{2}+3 c_{2} \kappa-b_{2} v-2 d_{2} \kappa v\right)-4 \kappa \delta_{1} \chi_{0}\left(\theta_{2}+\lambda_{2}+\beta_{2}+y_{2}\right)}{2 \eta_{3} d_{2}} t+\epsilon\right]} \\
\times & {\left[\sqrt{\delta_{1}\left(v_{1}-v_{2}\right)} \operatorname{csch}\left(\frac{1}{2} \sqrt{\frac{v_{1}-v_{2}}{\chi}}(x-v t)\right)\right] }
\end{aligned}
$$

where these solutions are valid for $\delta_{1}>0$. Moreover, if $\delta_{0}=-\delta_{1} v_{3}$ and $\zeta_{0}=0$, the Jacobi elliptic solutions (65) and (66) can be trimmed as

$$
\begin{aligned}
\mathfrak{p}(x, t)= & e^{i\left[-\kappa x+\frac{2 \eta_{3}\left(a_{1}+3 c_{1} \kappa-b_{1} v-2 d_{1} \kappa v\right)-4 \kappa \delta_{1} \chi_{0}\left(\theta_{1}+\lambda_{1}+\beta_{1}+y_{1}\right)}{2 \eta_{3} d_{1}} t+\epsilon\right]} \\
\times & {\left[\sqrt{\delta_{1}\left(v_{2}-v_{3}\right)} \operatorname{sn}\left(\mp \frac{1}{2} \sqrt{\frac{v_{1}-v_{3}}{\chi}}(x-v t), \frac{\left(v_{2}-v_{3}\right)}{\left(v_{1}-v_{3}\right)}\right)\right], } \\
\mathfrak{q}(x, t)= & e^{i\left[-\kappa x+\frac{2 \eta_{3}\left(a_{2}+3 c_{2} \kappa-b_{2} v-2 d_{2} \kappa v\right)-4 \kappa \delta_{1} \chi_{0}\left(\theta_{2}+\lambda_{2}+\beta_{2}+y_{2}\right)}{2 \eta_{3} d_{2}} t+\epsilon\right]} \\
\times & {\left[\sqrt{\delta_{1}\left(v_{2}-v_{3}\right)} \operatorname{sn}\left(\mp \frac{1}{2} \sqrt{\frac{v_{1}-v_{3}}{\chi}}(x-v t), \frac{\left(v_{2}-v_{3}\right)}{\left(v_{1}-v_{3}\right)}\right)\right] . }
\end{aligned}
$$

Remark 3. When $m \rightarrow 1$, dark soliton solutions emerge as

$$
\begin{aligned}
\mathfrak{p}(x, t) & =e^{i\left[-\kappa x+\frac{2 \eta_{3}\left(a_{1}+3 c_{1} \kappa-b_{1} v-2 d_{1} \kappa v\right)-4 \kappa \delta_{1} \chi_{0}\left(\theta_{1}+\lambda_{1}+\beta_{1}+y_{1}\right)}{2 \eta_{3} d_{1}} t+\epsilon\right]} \\
\times & {\left[\sqrt{\delta_{1}\left(v_{2}-v_{3}\right)} \tanh \left(\mp \frac{1}{2} \sqrt{\frac{v_{1}-v_{3}}{\chi}}(x-v t)\right)\right], } \\
\mathfrak{q}(x, t) & =e^{i\left[-\kappa x+\frac{2 \eta_{3}\left(a_{2}+3 c_{2} \kappa-b_{2} v-2 d_{2} \kappa v\right)-4 \kappa \delta_{1} \chi_{0}\left(\theta_{2}+\lambda_{2}+\beta_{2}+y_{2}\right)}{2 \eta_{3} d_{2}} t+\epsilon\right]} \\
\times & {\left[\sqrt{\delta_{1}\left(v_{2}-v_{3}\right)} \tanh \left(\mp \frac{1}{2} \sqrt{\frac{v_{1}-v_{3}}{\chi}}(x-v t)\right)\right] }
\end{aligned}
$$

for $v_{1}=v_{2}$. 


\section{Conclusion}

The work expounded in this article successfully that addresses optical solitons of Biswas-Arshed equation with Kerr-law nonlinearity in birefringent fibers with higher order dispersions and in the absence of fourwave mixing terms by the application of extended trial equation technique. With this integration scheme, we have commendably recovered dark, bright and singular optical solitons along with other traveling wave solutions, comprising rational solutions, periodic singular solutions and Jacobi elliptic function solutions in the presence of some constraints. It is concluded that our derived results for the Biswas-Arshed equation in birefringent fibers are exclusively new and have not been stated earlier. The outcomes of this paper are attention-grabbing and provide a stimulus to the audience of optical solitons. Later, this equation will be studied with the addition of four-wave mixing terms by the aid of appreciated integration schemes. These precious results will be presented as soon as possible.

Funding information: The authors state no funding involved.

Author contributions: All authors have accepted responsibility for the entire content of this manuscript and approved its submission.

Conflict of interest: The authors state no conflict of interest.

\section{References}

[1] Han SH, Park QH. Effect of self-steepening on optical solitons in a continuous wave background. Phys Rev E Stat Nonlin Soft Matter Phys. 2011 Jun;83(6 Pt 2):066601.

[2] Milovic D, Biswas A. Bright and dark solitons in optical fibers with parabolic law nonlinearity. Serb. J. Electr. Eng. 2013;10(3):365-70.

[3] Mirzazadeh M, Biswas A. Optical solitons with spatio-temporal dispersion by first integral approach and functional variable method. Optik (Stuttg). 2014;125(19):5467-75.

[4] Mirzazadeh M, Eslami M, Zerrad E, Mahmood MF, Biswas A, Belic M. Optical solitons in nonlinear directional couplers by sine-cosine function method and Bernoulli's equation approach. Nonlinear Dyn. 2015;81(4):1933-49.

[5] Manafian J, Lakestani M. Optical soliton solutions for the Gerdjikov-Ivanov model via $\tan (\phi / 2)$-expansion method. Optik (Stuttg). 2016;127(20):9603-20.
[6] Aziz UA, Attia S, Kashif AA, Muhammad O, Takasar H. Dynamical Aspects of Smoking Model with Cravings to Smoke. Nonlinear Eng. 2021;10(1):91-108.

[7] Ekici M, Mirzazadeh M, Sonmezoglu A, Ullah MZ, Asma M, Zhou Q, et al. Optical solitons with Schrödinger-Hirota equation by extended trial equation method. Optik (Stuttg). 2017;136:451-61.

[8] Aziz UA, Mashal A, Naeem U, Sohail N, Kashif AA. Thermal analysis of oblique stagnation point low with slippage on second-order fluid. J Therm Anal Calorim. 2021; https://doi.org/10.1007/s10973-021-10760-z.

[9] El-Borai MM, El-Owaidy HM, Ahmed HM, Arnous AH, Mirzazadeh M. Mirza-zadeh M. Solitons and other solutions to the coupled non-linear Schrodinger type equations. Nonlinear Eng. 2017;6(2):115-21.

[10] Yokuş A, Durur H, Abro KA. As?f Y, Hulya D, Kashif A A. Symbolic computation of Caudrey-Dodd-Gibbon equation subject to periodic trigonometric and hyperbolic symmetries. Eur Phys J Plus. 2021;136(4):358.

[11] Tahir M, Awan AU. Analytical solitons with Biswas-Milovic, equation in presence of spatio-temporal dispersion in non Kerr-law media. Eur Phys J Plus. 2019;134(9):464.

[12] Osman MS, Rezazadeh H, Eslami M. Traveling wave solutions for $(3+1)$-dimensional conformable fractional ZakharovKuznetsov equation with power law nonlinearity. Nonlinear Eng. 2019;8(1):559-67.

[13] Subasi M, Araz SI. Numerical regularization of optimal control for the coefficient function in a wave equation. Iran J Sci Technol Trans A Sci. 2019;43(5):2325-33. https://doi.org/10.1007/s40995-019-00690-9.

[14] Subasi M, Araz SI, Gungor H. On the numerical solution of two dimensional Schrodinger equation. Int J Math Res. 2017;6(1):1-12.

[15] Kashif AA, Abdon A, José FG. Role of bi-order Atangana-Aguilar fractional differentiation on Drude model: an analytic study for distinct sources. Opt Quantum Electron. 2021;53(4):177.

[16] Biswas A, Yildirim Y, Yasar E, Triki H, Alshomrani AS, Ullah $M Z$, et al. Optical soliton perturbation with full nonlinearity for Kundu-Eckhaus equation by modified simple equation method. Optik (Stuttg). 2018;157:1376-80.

[17] Qasim A, Samia R, Aziz UA, Kashif AA. A mathematical model for thermography on viscous fluid based on damped thermal flux. Zeitschrift für Naturforschung A, 2021; https://doi.org/10.1515/zna-2020-0322.

[18] Biswas A, Yildirim Y, Yasar E, Zhou Q, Moshokoa SP, Belic M. Optical solitons for Lakshmanan-Porsezian-Daniel model by modified simple equation method. Optik (Stuttg). 2018;160:24-32.

[19] Abro KA, Jose FG. Fractional modeling of fin on non-Fourier heat conduction via modern fractional differential operators. Arab J Sci Eng. 2021;46(3):2901-10.

[20] Biswas A, Yildirim Y, Yasar E, Zhou Q, Mahmood MF, Moshokoa SP, et al. Optical solitons with differential group delay for coupled Fokas-Lenells equation using two integration schemes. Optik (Stuttg). 2018;165:74-86.

[21] Syed TS, Abro KA, Sikandar A. Role of single slip assumption on the viscoelastic liquid subject to non-integer differentiable operators. Math Methods Appl Sci. 2021;44(7):1-16.

[22] Biswas A. Optical soliton perturbation with RadhakrishnanKundu-Lakshmanan equation by traveling wave hypothesis. 
Optik (Stuttg). 2018;171:217-20.

[23] Arshad R, Aziz UA, Sajad H, Sami UK, Abro KA. Effects of solid particles on fluid-particulate phase flow of non-Newtonian fluid through eccentric annuli having thin peristaltic walls. J Therm Anal Calorim. 2020; https://doi.org/10.1007/s10973020-10447-x.

[24] Arshed S, Biswas A, Abdelaty M, Zhou Q, Moshokoa SP, Belic M. Optical soliton perturbation for Gerdjikov-Ivanov equation via two analytical techniques. Zhongguo Wuli Xuekan. 2018;56(6):2879-86.

[25] Kashif AA. Numerical study and chaotic oscillations for aerodynamic model of wind turbine via fractal and fractional differential operators. Numer Methods Partial Differential Eq; 2020. pp. 1-15.

[26] Biswas A. Quasilinear pulses in birefringent fibers. Opt Quantum Electron. 2004;36(13):1167-86.

[27] Kashif AA, Sania Q, Abdon A. Mathematical and numerical optimality of non-singular fractional approaches on free and forced linear oscillator. Nonlinear Eng. 2020;9(1):449-56.

[28] Nathan PM, Kalyanasundaram N, Krishnam RM. Analysis of optical soliton propagation in birefringent fibers. Int. J. Microwave Opt. Technol. 2009;4(6):368-73.

[29] Dowluru RK, Bhima PK. Influence of third order dispersion on linear birefringent optical soliton transmission system. J Opt. 2011;40(3):132-42.

[30] Takasar H, Aziz UA, Abro KA, Muhammad O, Mehwish M. A mathematical and parametric study of epidemiological smoking model: a deterministic stability and optimality for solutions. Eur Phys J Plus. 2021;136(1):11.

[31] Girgis L, Milovic D, Konar S, Yildirim A, Jafari H, Biswas A. Optical Gaussons in birefringent fibers and DWDM systems with inter-modal dispersion. Rom Rep Phys. 2012;64(3):663-71.

[32] Imran QM, Abro KA, Muhammad AS, Asif AS. Functional shape effects of nanoparticles on nanofluid suspended in ethylene glycol through Mittage-Leffler approach. Phys Scr. 2020;96(2):025005.

[33] Tahir M, Awan AU, Rehman HU. Optical solitons to KunduEckhaus equation in birefringent fibers without four-wave mixing. Optik (Stuttg). 2019;199:163297.

[34] Yildirim Y, Biswas A, Zhou Q, Alshomrani AS, Belic MR. Sub pico-second optical pulses in birefringent fibers for KaupNewell equation with cutting-edge integration technologies. Results Phys. 2019;15:102660.

[35] Ozair M, Hussain T, Hussain M, Awan AU, Baleanu D, Abro KA A Mathematical and Statistical Estimation of Potential Transmission and Severity of COVID-19: A Combined Study of Romania and Pakistan. BioMed Res Int. 2020 Dec;2020:5607236.

[36] Ekici M, Sonmezoglu A. Optical solitons with Biswas-Arshed equation by extended trial function method. Optik (Stuttg). 2019;177:13-20.

[37] Kashif AA. Fractional characterization of fluid and synergistic effects of free convective flow in circular pipe through Hankel transform. Phys Fluids. 2020;32(12):123102.

[38] Yildirim Y. Optical solitons of Biswas-Arshed equation by trial equation technique. Optik (Stuttg). 2019;182:876-83.

[39] Zayed EM, Shohib RM. Optical solitons and other solutions to Biswas-Arshed equation using the extended simplest equation method. Optik (Stuttg). 2019;185:626-35.

[40] Tahir M, Awan AU, Rehman HU. Dark and singular optical solitons to the Biswas-Arshed model with Kerr and power law nonlinearity. Optik (Stuttg). 2019;185:777-83.

[41] Abro KA, Imran QM, Ambreen S. Thermal transmittance and thermo-magnetization of unsteady free convection viscous fluid through non-singular differentiations. Phys Scr. 2020;96(1):015215.

[42] Sabi'u J, Rezazadeh H, Tariq H, Bekir A. Optical solitons for the two forms of Biswas-Arshed equation. Mod. Phys. Lett. 2019;B33(25): 1950308. https://doi.org/10.1142/S0217984919503081.

[43] Kashif AA, Abdon A. Dual fractional modeling of rate type fluid through non-local differentiation. Numer Methods Partial Differential Eq; 2020:1-16.

[44] Tahir M, Awan AU. Optical singular and dark solitons with Biswas-Arshed model by modified simple equation method. Optik (Stuttg). 2020;202:163523.

[45] Tahir M, Awan AU. Optical travelling wave solutions for the Biswas-Arshed model in Kerr and non Kerr-law media. Pramana. 2020;94(1):29.

[46] Abro KA, Abdon A. Numerical and mathematical analysis of in- duction motor by means of AB-fractal-fractional differentiation actuated by drilling system. Numer Methods Partial Differential Eq; 2020:1-15.

[47] Yildirim Y. Optical solitons to Biswas-Arshed model in birefringent fibers using modified simple equation architecture. Optik (Stuttg). 2019;182:1149-62.

[48] Kashif AA, Ambreen S, Basma S, Abdon A. Application of statistical method on thermal resistance and conductance during magnetization of fractionalized free convection flow. Int Commun Heat Mass Transf. 2020;119:104971.

[49] Zayed EM, Alngar ME. Optical solitons in birefringent fibers with Biswas-Arshed model by generalized Jacobi elliptic function expansion method. Optik (Stuttg). 2020;203:163922.

[50] Awan AU, Tahir M, Rehman HU. Singular and bright-singular combo optical solitons in birefringent fibers to the BiswasArshed equation. Optik (Stuttg). 2020;210:164489.

[51] Tahir M, Awan AU. Optical dark and singular solitons to the Biswas-Arshed equation in birefringent fibers without fourwave mixing. Optik (Stuttg). 2020;207:164421.

[52] Rehman HU, Tahir M, Bibi M, Ishfaq Z. Optical solitons to the Biswas-Arshed model in birefringent fibers using couple of integration techniques. Optik (Stuttg). 2020;218:164894.

[53] Kashif AA, Mehwish S, Abdon A, Jose FG. Thermophysical properties of Maxwell Nanoluids via fractional derivatives with regular kernel. J Therm Anal Calorim. 2020; https://doi.org/10.1007/s10973-020-10287-9.

[54] Aziz UA, Samia R, Samina S, Abro KA. Fractional Modeling and Synchronization of Ferrouid on Free Convection Flow with Magnetolysis. Eur Phys J Plus. 2020;135(10):841-55.

[55] Abro KA, Bhagwan D. A scientific report of non-singular techniques on microring resonators: an application to optical technology. Optik (Stuttg). 2020;224:165696.

[56] Qasim A, Samia R, Aziz UA, Kashif AA. Thermal investigation for electrified convection flow of Newtonian fluid subjected to damped thermal flux on a permeable medium. Phys Scr. 2020;95(11):115003.

[57] Kashif AA. Role of fractal-fractional derivative on ferromagnetic fluid via fractal Laplace transform: A first problem via fractal-fractional differential operator. Eur J Mech BFluids. 2021;85:76-81. 
[58] Asf Y, Hulya D, Abro AK, Dogan K. Role of Gilson-Pickering equation for the different types of soliton solutions: A nonlinear analysis. Eur Phys J Plus. 2020;135(8):657.

[59] Aziz UA, Muhammad T, Kashif AA. Multiple soliton solutions with chiral nonlinear Schrödinger's equation in (2+1)dimensions. Eur J Mech BFluids. 2020;85:68-75.

[60] Kashif AA, Abdon A. Porous effects on the fractional modeling of magnetohydrodynamic pulsatile flow: an analytic study via strong kernels. J Therm Anal Calorim. 2020; https://doi.org/10.1007/s10973-020-10027-z.

[61] Abro KA, Abdon A. Numerical study and chaotic analysis of meminductor and memcapacitor through fractalfractional differential operator. Arab J Sci Eng. 2020; https://doi.org/10.1007/s13369-020-04780-4.

[62] Kashif AA, Atangana A. A comparative analysis of electromechanical model of piezoelectric actuator through CaputoFabrizio and Atangana-Baleanu frac- tional derivatives. Math Methods Appl Sci. 2020;43(17):1-11.

[63] Kashif AA, Jose FG. Role of Fourier sine transformon the dynamical model of tensioned carbon nanotubes with fractional operator. Math Methods Appl Sci. 2020:1-11.

[64] Kashif AA, Abdon A. Mathematical analysis of memristor through fractal-fractional differential operators: A numerical study. Math Methods Appl Sci. 2020;43(10):1-18.

[65] Aziz UA, Mukarram A, Abro KA. Electroosmotic slip flow of Oldroyd-B fluid between two plates with non- singular kernel. J Comput Appl Math. 2020;376:112885-99.

[66] Bhojraj L, Abro KA, Abdul WS. Thermodynamical analysis of heat transfer of gravity-driven fluid flow via fractional treatment: an analytical study. J Therm Anal Calorim. 2020;144(1):155-65.

[67] Kashif AA, Abdon A. A comparative study of convective fluid motion in rotating cavity via Atangana-Baleanu and CaputoFabrizio fractal-fractional differentiations. Eur Phys J Plus. 2020;135(2):226-42.

[68] Kashif AA, Ambreen S, Abdon A. Thermal stratification of rotational second-grade fluid through fractional differential operators. J Therm Anal Calorim. 2020;143(5):3667-76.

[69] Abro KA, Abdon A. Role of non-integer and integer order differentiations on the relaxation phenomena of viscoelastic fluid. Phys Scr. 2020;95(3):035228.

[70] Abro KA. A fractional and analytic investigation of thermodiffusion process on free convection flow: an application to surface modification technology. Eur Phys J Plus. 2020;135(1):31-45.

[71] Ambreen S, Kashif AA, Muhammad AS. Thermodynamics of magnetohydrodynamic Brinkman fluid in porous medium: applications to thermal science. J Therm Anal Calorim. 2019;136(6):2295-304.

[72] Kashif AA, Irfan AA, Sikandar MA, Ilyas K. On the thermal analysis of magnetohydrodynamic Jeffery fluid via modern non integer order derivative. J King Saud Univ Sci. 2019;31(4):973-9. 